Pedagogía y Saberes No. 49

Universidad Pedagógica Nacional

Facultad de Educación. 2018, pp. 165-176

\title{
El imperativo de la inclusión por habitabilidad: el sujeto aprendiente ${ }^{*}$
}

Artículo de investigación

The Imperative of Inclusion by Habitability: The

Learning Subject

O imperativo da inclusão por habitabilidade: o sujeito

aprendente

David Rubio-Gaviria**

Kamila Lockmann ${ }^{* * *}$

Para citar este artículo:

Rubio-Gaviria, D. y Lockmann, K. (2018). El imperativo de la inclusión por habitabilidad: el sujeto aprendiente. Pedagogía y Saberes, 49, 165-176.

* Una primera versión de este texto fue presentada por sus autores en el V Coloquio Latinoamericano de Biopolítica, llevado a cabo en septiembre del 2015 en la Universidade do Vale do Rio dos Sinos (Unisinos), Brasil. Agradecemos el trabajo de traducción al español de esa primera versión a la profesora Yeraldin Quimbayo Lozano, profesional de la Secretaría de Educación de Bogotá.

** Profesor de la Facultad de Educación de la Universidad Pedagógica Nacional. Doctor en Educación e investigador del Grupo de Historia de la Práctica Pedagógica-GHPP.

Correo electrónico: drubio@pedagogica.edu.co

Código ORCID: http://orcid.org/0000-0002-4102-8267

*** Profesora de la Universidade Federal do Rio Grande-FURG. Doctora en Educación e investigadora del grupo de Estudos e Pesquisa em Inclusão-GEPI/UNISINOS/CNPQ, del grupo Grupo de Estudos e Pesquisas em Currículo e Pós-modernidade -GEPCPÓs da UFRGS e do Grupo de Estudo e Pesquisa em Inclusão - GEPI da UNISINOS.

Correo electrónico: kamila.furg@gmail.com

Código ORCID: http://orcid.org/0000-0002-1993-8088 


\title{
Resumen
}

Este artículo de reflexión se propone mostrar la utilidad de las herramientas genealógicas y arqueológicas de Michel Foucault para la investigación en educación. Para ello, se analiza la inclusión en tanto imperativo de los procesos educativos contemporáneos, desde la perspectiva de la investigación que nos ofrecen las herramientas del filósofo francés, específicamente la de gubernamentalidad. El artículo presenta, en primer lugar, una caracterización sobre los alcances y los límites del trabajo con inspiración genealógica, como posibilidad para la investigación en educación, en segundo lugar, una lectura de los procesos de in/exclusión, con principal énfasis en la Modernidad; y finalmente, algunas posibilidades para pensar el imperativo contemporáneo de la inclusión, como un asunto que llamamos inclusión por habitabilidad, que tiene como característica fundamental la concepción del aprendizaje como posibilidad para la inclusión en las llamadas ciudades educativas.

\section{Palabras clave}

inclusión por habitabilidad; genealogía; gubernamentalidad; investigación educativa; aprendizaje

\begin{abstract}
This reflection paper aims to show the usefulness of Michel Foucault's genealogical and archaeological tools for research in education. To that end, we analyze the inclusion as imperative of contemporary educational processes, from the perspective of research offered by the tools of the French philosopher, specifically that of governmentality studies. First, the paper presents a characterization of the scope and limits of work with a genealogical inspiration as a possibility for research in education. Secondly, the paper proposes a reading of the processes of in/exclusion, with the main emphasis on modernity. Finally, the paper presents some possibilities to think about the contemporary imperative of inclusion, as an issue that we call habitability for inclusion, which has the fundamental characteristic of the conception of learning as a possibility for inclusion in the so-called educational cities.
\end{abstract}

\section{Keywords}

Inclusion by habitability; genealogy; governmentality; educational research; learning

\section{Resumo}

Este artigo de reflexão visa mostrar a utilidade das ferramentas genealógicas e arqueológicas de Michel Foucault para a pesquisa em educação. Para isso, analisamos a inclusão, entendido como um imperativo dos processos educativos contemporâneos, desde a perspectiva de pesquisa oferecida pelas ferramentas do filósofo francês, especificamente, a governamentalidade. 0 artigo apresenta, em primeiro lugar, uma caracterização sobre os alcances e limites do trabalho de inspiração genealógica, como possibilidade para a pesquisa em educação; em segundo lugar, uma leitura dos processos de in/exclusão, com principal ênfase na Modernidade; e finalmente, algumas possibilidades para pensar o imperativo contemporâneo da inclusão, o qual denominamos inclusão por habitabilidade, que tem como característica fundamental a concepção da aprendizagem como possibilidade para a inclusão nas chamadas cidades educativas.

\section{Palavras chave}

inclusão por habitabilidade; genealogia; governamentalidade; investigação educativa; aprendizagem 


\section{Introducción}

El texto presenta algunas discusiones acerca de las nociones de inclusión y aprendizaje, mostrando cómo la articulación entre ellas es central hoy en nuestros países: Brasil y Colombia. A partir de una investigación bibliográfica apoyada, sobre todo, en el pensamiento de Michel Foucault, comprendemos que las prácticas de inclusión (y también de exclusión) son, por excelencia, prácticas de gobierno que operan de formas distintas sobre la conducta de los sujetos. En este texto argumentamos que, en la contemporaneidad, es posible observar nuevas formas de gobierno que no operan tanto por medio de la exclusión de los sujetos como en la Edad Media, o de la reclusión en instituciones de confinamiento como en la Modernidad, sino por medio de prácticas de inclusión que priorizan la circulación de los sujetos por el espacio abierto. Esas nuevas formas de gobierno se fundamentan en lo que aquí denominamos inclusión por habitabilidad ${ }^{1}$ y exigen nuevas estrategias de inversión en los sujetos y en la regulación de su conducta, las cuales son desarrolladas por medio de procesos de aprendizaje constantes y permanentes. Así, pretendemos sostener la tesis según la cual la emergencia de la inclusión por habitabilidad como un imperativo de nuestro tiempo solo ocurre a partir del momento en que la noción de aprendizaje asume un papel central en nuestras sociedades. De esta forma, podemos decir que las prácticas de inclusión por habitabilidad - que proliferan en la actualidad - necesitan del aprendizaje como elemento clave para su funcionamiento.

Todo eso, claro está, se desarrolla al interior de una racionalidad política, económica y social que podemos denominar neoliberal, la cual asume a los sujetos como económicamente activos y, por eso mismo, responsables por las inversiones que deben permanentemente hacer en sí mismos, en sus habilidades y competencias. Volveremos a estas discusiones más adelante.

Dado que se trata de un asunto central para la educación, tal como se vive en nuestro presente, proponemos lanzar una mirada genealógica sobre los desplazamientos operados en las prácticas de exclusión e inclusión desarrolladas en Occidente, intentando comprender la forma como fueron produciendo ciertos modos particulares de vivir la inclusión y la exclusión en la actualidad.

Antes de ello, por supuesto, es necesario explicar el modo en que operamos metodológicamente con el

1 Concepto creado por los autores y se desarrollará más adelante en el artículo. concepto-herramienta de la genealogía. Tales discusiones son presentadas en la primera sección.

En la segunda sección pasamos por tres momentos distintos: la Antigüedad y la Edad Media, en las que observamos el desarrollo de prácticas de exclusión por medio de la eliminación o de la segregación del anormal; la Modernidad, a partir de la cual abordaremos las prácticas de inclusión por reclusión, es decir, los procesos de institucionalización de los anormales desarrollados por la sociedad disciplinar; $y$, finalmente, un tercer momento en el que detendremos el análisis en la contemporaneidad, tematizando las prácticas de inclusión por habitabilidad, las cuales exigen nuevas inversiones en los sujetos por medio de aprendizajes permanentes.

En una tercera sección, situados en lo contemporáneo, desarrollamos el argumento según el cual es posible comprender la inclusión como un imperativo de nuestro tiempo, es decir, como algo que se impone a cada uno de nosotros, una verdad que nos subjetiva y nos hace ejercer, sobre los otros y sobre nosotros mismos, determinadas acciones que conducen nuestras conductas. Sin embargo, no podemos hablar del imperativo de la inclusión de un modo genérico u homogéneo. Comprendemos que, en la racionalidad neoliberal, observamos un tipo muy específico de inclusión que, al articularse profundamente con la noción de aprendizaje, funciona por medio de lo que denominamos habitabilidad. De este modo, no es la inclusión la que se constituye en un imperativo contemporáneo, sino la inclusión por habitabilidad.

\section{Una inspiración genealógica para revisar la historia}

\author{
Necesitamos de una consciencia histórica de la \\ situación del presente.
}

FouCAULT, 2010, p. 232

¿Por qué, en una sociedad y una época como la nuestra, existe un número considerable de programas y proyectos de inclusión escolar y social? ¿Qué explica esa expansión y proliferación en la actualidad? ¿Desde cuándo? ¿Qué posibilitó la constitución de esas prácticas en nuestras sociedades? En suma, ¿cuál es la procedencia y las condiciones de emergencia de las prácticas de in/exclusión que se configuran en nuestro presente?

Para responder tales cuestiones, proponemos una mirada genealógica sobre la emergencia de lo 
que denominamos inclusión por habitabilidad, y sobre algunas prácticas de inclusión y exclusión que se desenvolvieron a lo largo de la historia de Occidente y funcionaron como condición de posibilidad para la constitución del presente. Para ello, tomamos prestado de Nietzsche y Foucault el concepto de genealogía: un tipo de análisis histórico que se opone a la pregunta por el origen (Ursprung). La investigación genealógica no pretende revelar un punto primero originario, del cual todo evolucionó. No se trata de mostrar la esencia de determinado objeto o de desvelar, en el pasado, un punto embrionario donde estarían alojados determinados conceptos e ideas. Según Foucault (2010, p. 17), "buscar tal origen es intentar reencontrar lo que 'era inmediatamente', o 'aquello mismo' de una imagen exactamente adecuada a sí [...] es querer retirar las máscaras para revelar, en fin, una identidad primera". Para el autor, la investigación sobre el origen se constituye en un proyecto metafísico que busca el momento y el lugar donde las cosas se encontraban en un estado de perfección. Foucault, al igual que Nietzsche, rechaza la investigación sobre el origen y, en lugar de creer en la metafísica, propone que interroguemos a la historia, sus agitaciones, discordias, sorpresas, desvíos y accidentes. Se trata de comprender que la constitución de un objeto (y de prácticas) es el resultado del cruce de una variedad de prácticas y discursos que tornaron posible su existencia en un momento histórico dado. Para Foucault (2010, p. 21),

Seguir el hilo complejo de la procedencia es [...] mantener lo que pasó en la dispersión que le es propia: es demarcar los accidentes, los ínfimos desvíos - $\mathrm{o}$ al contrario las inversiones complejaslos errores, las fallas en la apreciación, los malos cálculos que dieron nacimiento a lo que existe y tiene valor para nosotros; es descubrir que en la raíz de aquello que conocemos y aquello que somos no existe en la verdad y el ser, sino en la exterioridad del accidente.

Así, no se trata de desvelar un único comienzo, sino de buscar en la historia, en el acontecer mismo de la vida social, las variadas y las multifacéticas condiciones que posibilitaron la constitución ${ }^{2}$ de un conjunto de prácticas vinculadas a lo que llamamos "inclusión", con sus estrategias y sus propios objetos.

2 Utilizamos la expresión variadas y multifacéticas condiciones de posibilidad porque hablar apenas de diferentes condiciones parece reducir la complejidad de la imbricación de prácticas que contribuyen para la aparición de un dado objeto en la historia. Así, más allá de diferentes, se trata de variadas y multifacéticas condiciones de posibilidad que, incluso situándose en esferas distintas de la vida social, se articulan/ relacionan para engendrar nuevas "cosas"/realidades, etc. (Veiga-Neto, 1996).
Una mirada genealógica presupone considerar una serie de hechos que, al estar relacionados, pueden haber contribuido para su emergencia. Eso evita la búsqueda incesante de la historia totalizante, relatando, cronológicamente, todos los acontecimientos que se relacionan con la constitución de las prácticas de in/exclusión en nuestros países. Conforme destaca Veyne (1998, p. 18), “[...] en ningún caso, lo que los historiadores llaman evento es aprehendido de manera directa y completa [...] es siempre incompleta y lateral, por documentos, testimonios, o sea, por tekmeria, por indicios".

Aprendemos que para cualquier forma de contar una historia se realiza un proceso de selección, de inclusión de algunos acontecimientos y de exclusión de otros. La historia nunca consigue y, en ese caso, no pretende ser totalizante. Ella selecciona, organiza, produce recortes, destaca determinadas escenas y suprime otras, como señala Bauman:

Las historias son como reflectores -iluminan partes del palco en cuanto dejan el resto en la oscuridad-. Si iluminasen igualmente el palco en conjunto, de hecho no tendrían utilidad [...]. Es misión de las historias seleccionar, y es de su naturaleza incluir excluyendo e iluminar lanzando sombras. Es un grave error, además de ser una injusticia, culpar a las historias por favorecer una parte del palco y suprimir otra. (2005, p. 26).

Por esa razón, no tenemos la intención o la pretensión de contar una historia totalizante, relatando cronológicamente todos los acontecimientos que se relacionan con los procesos de in/exclusión. En ese proceso de elecciones (de iluminación sobre una parte del palco), optamos por realizar el siguiente ejercicio metodológico: intentamos comprender cómo, en cada momento histórico específico, las prácticas de inclusión y exclusión se vinculan a determinadas verdades de ese tiempo, produciendo modos distintos para conducir las conductas de los sujetos y de las poblaciones. Son, por tanto, formas de gobierno que se muestran relacionadas con determinadas formas de manifestación de la verdad. Con Foucault aprendemos que

[...] el ejercicio de poder se acompaña constantemente de una manifestación de la verdad entendida en un sentido amplio [...] se podría llamar la manifestación de la verdad como un conjunto de procedimientos posibles, verbales o no, por los cuales se actualiza eso que es colocado como verdadero. (2010, p. 35).

Podemos decir que, en cada época estudiada, tenemos una forma de manifestación de la verdad, o sea, un conjunto de procedimientos que producen, 
por medio de una ritualización, algo como siendo verdadero que, por eso mismo, muestra una fuerza imponente. Lo que se impone a los individuos no es la forma de manifestación de la verdad, sino su propio régimen de verdad, al cual esa manifestación se encontraba vinculada. El régimen de verdad puede ser comprendido, siguiendo a Foucault (2010, p. 67), como "aquello que impone a los individuos un cierto número de actos de verdad [...] aquello que define, que determina la forma de esos actos; es aquello que establece para esos actos condiciones y efectos específicos"; es el régimen de verdad, por lo que él expresa de verdadero, que muestra fuerza de imposición y que hace que el individuo diga: "si es verdadero, ¡me inclinaré! Si es verdad, por tanto, ¡me inclino!” (Foucault, 2010, p. 71). Tal operación hace que el sujeto conduzca sus propias acciones a partir de determinados regímenes de verdad; pasa a ser subjetivado por esos regímenes. No se trata aquí de actos de obediencia o sumisión, sino de actos de verdad mediante los cuales mostramos nuestro reconocimiento y aceptación de algo como verdadero.

De esa manera, es posible comprender que, en cada época histórica abordada en este texto, las prácticas de in/exclusión aparecen vinculadas a un régimen de verdad específico que se impone a los individuos y los lleva a ejercer determinados actos de verdad que modelan sus conductas y sus relaciones con los otros. En la actualidad, tenemos un régimen de verdad pautado en el discurso de la inclusión, que se constituye en un imperativo. Por eso, lo que pretendemos mostrar aquí es que no se trata de cualquier tipo de inclusión, sino de una que prevé la circulación de los sujetos por el espacio abierto de la ciudad, pues justamente lo que constituye las condiciones de su inclusión es su posibilidad de habitar ciudades que, en su modo de ser moderno, están caracterizadas por ser espacios que privilegian la circulación de quienes las habitan, en el sentido en que Sennett (1994) ha comprendido este fenómeno. El imperativo contemporáneo no es simplemente la inclusión, sino la inclusión por habitabilidad.

Una mirada genealógica sobre la historia de esas prácticas de in/exclusión nos ayuda a construir un camino posible para comprender las condiciones de posibilidad de esa inclusión por habitabilidad en el presente. Es esa empresa la que pretendemos desarrollar en la siguiente sección.

\section{Desplazamientos históricos en las prácticas de in/ exclusión: de la Antigüedad a la contemporaneidad}

Como podemos percibir, en diferentes momentos históricos se buscó identificar y marcar aquellos individuos que no encajaban en los patrones de normalidad. Podemos decir, entonces, que la anormalidad siempre fue una preocupación social y política, lo que difiere, en cada época, son las prácticas desarrolladas para gobernarla.

En la Antigüedad y en la Edad Media son rastreables prácticas de exclusión que acontecían bien por exterminio, eliminación y muerte, bien por segregación, distanciamiento y expulsión. Varios estudios han demostrado que la anormalidad era, en ese periodo, explicada por fuerzas transcendentales que la veían como objeto de maldición o como divinidad. En la Antigüedad Clásica, en Esparta, Atenas y Roma, los niños que nacían deformes eran llevados a un lugar secreto fuera de la ciudad para dejarlos morir o para ser ahogados (Benvenuto, 2006). Eran prácticas de exterminio y muerte como las que podemos observar en las herejías del siglo XII, las cuales eran combatidas por la Inquisición, una práctica de la Iglesia católica para arrancar el demonio del cuerpo por medio de una purificación por las llamas. Sin embargo, además de esas prácticas de exclusión por eliminación podemos encontrar, aún en la Edad Media, prácticas de exclusión por distanciamiento o desaparición social de aquellos que eran considerados anormales. Veamos dos de esas prácticas presentadas por Michel Foucault: en primer lugar, el caso de los leprosos en la Edad Media, descrito por Foucault en el célebre curso "Los anormales":

Todo el mundo sabe cómo se desarrollaba a fines de la Edad Media, e incluso en todo el transcurso de ésta, la exclusión de los leprosos. La exclusión de la lepra era una práctica social que implicaba, en principio, una partición rigurosa, una puesta a distancia, una regla de no contacto entre un individuo (o un grupo de individuos) y otro. Se trataba, por otra parte, de la expulsión de esos individuos hacia un mundo exterior, confuso, más allá de las murallas de la ciudad, más allá de los límites de la comunidad. (Foucault, 2007c, p. 50).

Tales prácticas declaraban la muerte simbólica del leproso por medio de rituales fúnebres y de transferencia de sus bienes a otros. El leproso era llevado a un mundo exterior, nadie más se interesaría por su paradero, nadie más tendría contacto con él, o estaría 
esperando su retorno después de la cura. En realidad, su "muerte" había sido consumada.

La segunda práctica de exclusión descrita por Foucault se refiere a la nave de los locos o de los insensatos, tematizada en su libro Historia de la locura:

Confiar el loco a los marineros es evitar, seguramente, que el insensato merodee indefinidamente bajo los muros de la ciudad, asegurarse de que irá lejos y volverlo prisionero de su misma partida. Pero a todo esto, el agua agrega la masa oscura de sus propios valores; ella lo lleva, pero hace algo más, lo purifica; además, la navegación libra al hombre a la incertidumbre de su suerte; cada uno queda entregado a su propio destino, pues cada viaje es, potencialmente, el último. [...] El agua y la navegación tienen por cierto este papel. Encerrado en el navío de donde no se puede escapar, el loco es entregado al río de mil brazos, al mar de mil caminos, a esa gran incertidumbre exterior a todo. Está prisionero en medio de la más libre y abierta de las rutas. (2015, p. 26).

Como vemos, tales prácticas de exclusión se desarrollaron por medio del distanciamiento y de la desaparición social de todos aquellos que de alguna forma amenazaban el orden y la tranquilidad de la ciudad. Durante toda la Edad Media la estrategia de defensa social frente al problema de las anormalidades fue siempre la exclusión por exterminio o por distanciamiento. Tales prácticas ocurrían porque en la época no había un saber sobre esas anormalidades. Esos sujetos eran, por así decir, extraños. Siendo tal, la única forma de ejercer el poder sobre ellos consistía en una división binaria que decidía sobre el derecho de vida y sobre el derecho de muerte de esos individuos (aunque fuese una muerte simbólica). He aquí el ejercicio del poder soberano, de ostentación y de espectáculo, que ejercido en los límites de un territorio, con el propósito de purificación y limpieza de la ciudad, era un poder de espada, que solo decidía sobre la vida de esos sujetos justamente porque podía decidir sobre su muerte. Era, por tanto, un poder que "hacía morir y dejaba vivir" (Foucault, 2001).

Hasta este momento, vemos, mediante esos procesos de exclusión, una tecnología de poder que expulsa, excluye, elimina, distancia, margina; es un poder que se expresa por medio de sus formas negativas. Sin embargo, entre finales del siglo xviI y el inicio del siglo XVIII, observamos la emergencia de una nueva forma de poder,

[...] un poder que es por fin un poder positivo, un poder que fabrica, que observa, un poder que sabe y se multiplica a partir de sus propios efectos. [...] Un poder que no actúa por la separación en grandes masas confusas, sino por distribución según individualidades diferenciales. Un poder que no está ligado al desconocimiento sino, al contrario, a toda una serie de mecanismos que aseguran la formación, la inversión, la acumulación, el crecimiento del saber. (Foucault, 2007b, p. 55).

Para ejemplificar esa nueva tecnología de poder, Foucault aborda el modelo de la peste, a partir del cual podemos observar una serie de regulaciones que solo pudieron existir a partir del momento en que se construye un saber sobre la peste y sus formas de contagio. Con ese conocimiento fue posible cuadricular, literalmente, la ciudad en "estado de peste" y acompañar, vigilar y regular los hábitos de las personas, evitando determinados contactos. Se puede decir que la preocupación se dirigía al cuerpo individual, a una vigilancia exhaustiva sobre cada habitante de la ciudad y un control por medio de registros que permitían gestionar el riesgo causado por la peste. No se trataba de excluir, sino de incluir para distribuir, para establecer una red de miradas, un mecanismo de visibilidad general, controlando a cada uno individualmente. No era tampoco un rito de purificación, ni siquiera un ejercicio de poder que produce la muerte o la desaparición del cuerpo anormal, sino que era, en el caso de la peste, un intento de maximizar la salud, la vida, la longevidad, la fuerza de los individuos, por tanto, de hacer vivir, aunque actuando en ese momento, bajo el ámbito individual.

Se puede percibir allí la emergencia de una forma diferente de poder, uno que actúa sobre la vida, que Foucault (2007b) denominó biopoder. Según el autor, este se presenta mediante dos formas principales: una anatomopolítica del cuerpo humano, que surge en el siglo xvir y opera sobre el cuerpo individual, y una biopolítica de la especie humana, que se consolida a mediados del siglo XVIII e interviene sobre el ámbito colectivo, sobre la población como conjunto con regularidades. Lo que es preciso comprender es que, ya en el siglo XviI, ese poder que actúa sobre el cuerpo individual se organiza en torno a la vida y es, por tanto, un biopoder, una primera cara de este. Es en ese primer aspecto del poder sobre la vida que encontramos el modelo de la peste.

Dicho modelo implicó una primera operación del poder en la sociedad disciplinar. Sin embargo, en este, el sujeto pestífero permanecía en su propia casa, lo que demandaba de una vigilancia exhaustiva, y un control muy amplio y minucioso. Por una cuestión de economía del poder, surgen las instituciones disciplinares y no solo el pestífero y el leproso son institucionalizados, sino todo sujeto que pudiera constituirse como una amenaza para la población. 
Aquí la estrategia de defensa social no es más la exclusión, sino la inclusión, la cual va a caracterizar la Modernidad o la llamada sociedad disciplinar. La inclusión fue una invención de la Modernidad con el objetivo de poner en orden la sociedad, civilizar, disciplinar y gobernar. Sin embargo, no tomemos el término inclusión como un concepto univoco: posee alcances muy heterogéneos. Para este periodo, nos referimos a un tipo de inclusión ejercida mediante la reclusión y la institucionalización de esos individuos, por su localización y distribución en aparatos de saber. La nave de los locos "no irá de un más acá del mundo a un más allá, en su tránsito extraño [...] ahora ha atracado entre las cosas y la gente. Retenida y mantenida, ya no es barca, sino hospital" (Foucault, 2015, p. 73). A esta podemos añadir otras instituciones como el cuartel, la escuela, la prisión, la fábrica, los manicomios, las casas de acogimiento a la infancia y la juventud vulnerable, entre tantos otros establecimientos de encierro del cuerpo que emergieron en la Modernidad. El cuerpo anormal es institucionalizado y sometido a un aparato de poder/saber que fija a los individuos en determinados espacios, controla la utilización del tiempo y regula las acciones de cada uno, enseñando formas de actuar y comportarse que serán útiles para el gerenciamiento de la sociedad.

Analizando tales procesos de institucionalización, no podemos dejar de mencionar la escuela y la forma como surge, en nuestra sociedad, mucho más como una maquinaria de gobierno de la conducta, que como una institución de enseñanza. La escuela siempre fue ese mecanismo de gobierno y control de los vivientes, lo cual se debe al hecho de que todas las prácticas educativas, independientemente de sus inclinaciones ideológicas, políticas, metodológicas o teóricas, son, por excelencia, prácticas de gobierno, de conducción de las conductas (Noguera, 2011).

La producción de esos modos de vida adecuados a la sociedad comenzó a ser operada no solo sobre los individuos normales que ya eran blanco de las técnicas educativas, sino también sobre los anormales que pasaron a frecuentar y a habitar los espacios educativos institucionalizados. El cuerpo anormal es capturado por la escuela y por otras instituciones disciplinares que produjeron una serie de regulaciones sobre él, enseñando modos de ser, vivir y conducirse en el mundo. Un primer movimiento de institucionalización de esos individuos en los espacios escolares ocurrió al final del siglo XIX con la creación de los primeros institutos especializados para la atención de niños ciegos y sordos. Muchos estudiosos han comprendido esas prácticas como un movimiento de segregación de los sujetos anormales, pues se encontrarían aislados de los demás. En lugar de eso, proponemos pensar esas prácticas como un primer movimiento de inclusión de los sujetos anormales en los procesos educativos, una inclusión que se da por medio de la reclusión.

Sin embargo, la noción de inclusión fue sufriendo modificaciones y se fue adecuando a las necesidades contemporáneas. Poco a poco durante el siglo xx, otras formas de inclusión fueron estructurándose y pueden ser localizadas tanto en Brasil como en Colombia, a partir de la década de 1990, cuando la inserción de los sujetos deficientes en las escuelas regulares comienza a producirse mediante políticas educativas. En el momento en que esos sujetos pasan a frecuentar y circular en las escuelas regulares y también en la sociedad de una forma más amplia, percibimos que no basta apenas con actuar sobre los anormales, que parecían ser el foco hasta entonces, sino que también se pasa a desarrollar una serie de intervenciones sobre los normales para que pudieran aceptar, respetar y producir un medio ambiente adecuado y capaz de asegurar la presencia, la participación y la circulación de todos. De esa forma, ya no estamos frente a una inclusión que actúa por medio de la reclusión de los individuos en espacios cerrados, sino que se guía por el principio de habitabilidad, es decir, produce determinadas condiciones para que esos individuos puedan habitar el espacio abierto tanto de la escuela ${ }^{3}$ como de la ciudad. La aparición de esta nueva noción -inclusión por habitabilidadocurre de forma inmanente a las modificaciones sociales que sacudieron las estructuras disciplinares en boga hasta entonces.

Aunque la disciplina y, obviamente, las instituciones disciplinares no hayan desaparecido, parecen estar sufriendo un claro proceso de reconfiguración. La escuela, por ejemplo, ha sido convocada como instancia de solución y resolución de una variedad de problemas sociales, y responsabilizada por el subsecuente desarrollo de múltiples proyectos y programas que han producido una considerable ampliación de sus funciones. Con esto, también vemos ampliar el espectro de actuación de la escuela que extrapola el límite de la institución escolar. Por más que tales proyectos sean desarrollados, la mayoría de las veces, al interior de la escuela, su alcance presenta una amplitud que va mucho más allá de los muros de la institución escolar, diseminándose por los más amplios sectores de la vida social. Del mismo modo, una multiplicidad de aspectos sociales, económicos,

3 Las discusiones en materia de política educativa que indican la necesidad de replantear a la escuela como un espacio abierto son rastreables desde los años setenta (véase Faurè, 1973). Así mismo, es posible localizar discusiones en los años noventa en Brasil y Colombia a este respecto. 
culturales y empresariales ha invadido la escuela en los últimos años, produciendo también grandes modificaciones en sus aparatos disciplinares.

Esa nueva configuración de la escuela está encuadrada con la lógica contemporánea. Actualmente, parece que estamos viviendo una especie de crisis de los dispositivos disciplinares, tal como anunciaba Deleuze (1990) al abordar el surgimiento de las sociedades de control. El filósofo planteaba que estamos frente a una crisis generalizada de todos los medios de confinamiento y que las formas de dominación ya no son ejercidas por medio de instituciones como la escuela, la fábrica, el hospital o la prisión, sino que presentan un modus operandi completamente distinto. En la actualidad no es suficiente y ni siquiera necesario capturar los cuerpos anormales, encuadrarlos e institucionalizarlos para regular sus acciones, sino que tal regulación debe ocurrir en el espacio abierto por medio de la modulación de la subjetividad de todos y cada uno.

Así, los sujetos anormales no son más prisioneros del viaje, como en la nave de los insensatos, y mucho menos se encuentran encarcelados en las instituciones de encierro inventadas en la Modernidad. En la contemporaneidad, la barca naufraga y se producen grietas en los muros de las instituciones disciplinares de confinamiento. Por medio de la inclusión que se ejerce por la habitabilidad de todos en un espacio abierto de la ciudad, otras formas de gobierno son ejercidas y pueden ser ampliamente identificadas en nuestro presente.

Es en este proceso que el aprendizaje se torna central en nuestro presente, pues la inclusión por habitabilidad solo puede ocurrir por medio de inversiones que son realizadas sobre los sujetos normales $\mathrm{y}$ anormales, las cuales permiten que construyan determinados aprendizajes para poder habitar el espacio abierto de la ciudad sin constituirse en un riesgo para sí o para los demás. Así, podemos decir que las nuevas formas de gobierno contemporáneo actúan por medio de la inclusión, pero también del aprendizaje permanente. Estas dos nociones, articuladas, logran poner en funcionamiento aquello que denominamos inclusión por habitabilidad, concepto que a continuación nos proponemos examinar.

\section{Aprendizaje, gobierno y habitabilidad}

¿Cómo el discurso de la inclusión se constituye en un imperativo en la actualidad? Cuando hablamos de inclusión hoy, ¿a qué nos estamos refiriendo? ¿Cómo funciona la inclusión y cómo se articula con la educación para el gobierno de los sujetos tanto normales como anormales? ¿Quiénes son los sujetos que están al margen de la sociedad en este imperativo de la inclusión?

Antes que responder estas preguntas, más bien proponemos algunas posibilidades para pensar la inclusión y el aprendizaje como nuevas formas de gobierno de la sociedad contemporánea. Partimos de una discusión inicial sobre la centralidad que la noción de aprendizaje tiene hoy, para posteriormente retomar la noción de inclusión y su funcionamiento articulado con aquella, lo que nos permite proponer el concepto que interesa: la inclusión por habitabilidad.

Nuestra hipótesis para el final de este texto parte del presupuesto de que estamos viviendo un desplazamiento en la conducción de las conductas y en la pedagogización de lo social: de la enseñanza hacia el aprendizaje. Sobre eso, Noguera (2011, p. 230) afirma que

\section{[...] si los conceptos de doctrina y disciplina go- bernaron las reflexiones pedagógicas durante la Edad Media; si institutio y eruditio dominaron el pensamiento pedagógico de los siglos XVI y XVII; si educación, instrucción y Bildung prevalecieron entre el final del siglo xvir y el final del xIX, el con- cepto de aprendizaje (learning), será el concepto pedagógico preponderante del siglo $\mathrm{xx}$, así como de los inicios del XXI.}

La aparición del concepto "aprendizaje" está asociada tanto a la biología y a las teorías evolucionistas del siglo XIX, como a las llamadas psicologías del aprendizaje de inicio del siglo $\mathrm{xx}$, e incluso a las discusiones de la filosofía moral y política, y la economía durante la segunda mitad del siglo XIX y al menos las primeras tres décadas del xx (Rubio, 2017). La Escuela Nueva y, fundamentalmente, la psicología buscaron desarrollar una especie de psicologización de los niños por el imperativo de la actividad, el crecimiento y el desarrollo en el escenario del aprendizaje. De este modo,

Con la aparición del concepto de aprendizaje, la concepción de la educación fue transformada. El énfasis en la formación del carácter, esto es, en la función moral de la educación y de la instrucción, concebida por la mayor parte de los pedagogos del siglo XIX, va cambiando en los inicios del siglo $\mathrm{XX}$ en la dirección de nuevos procesos de crecimiento, desarrollo o adaptación del individuo (organismo) a su medio, constituyendo así una especie de moral biológica. (Noguera, 2011, p. 242).

Esta moral biológica puede ser leída como un proceso por el cual el equipamiento humano (organismo, 
individuo) debe ser llevado a adaptarse, a ajustarse al medio (ambiente, ciudad) y vivir en él (habitarlo) de forma productiva. Comprendemos que esos procesos de adaptación no son naturales y requieren de la educación para efectuarse. Sin embargo, esta aquí no se rige tanto por las intervenciones realizadas sobre los individuos directamente (enseñanza), sino por aquellas intervenciones que actúan sobre el medio y que organizan el ambiente para, a partir de allí, ejercer una acción sobre los sujetos.

Para comprender las cosas de forma más clara, basta observar las propuestas del movimiento de la Escuela Nueva, en el que pensadores como María Montessori, por ejemplo, organizaban el espacio, es decir el medio, para que en la relación con él los niños activos pudieran desarrollar sus aprendizajes. No se trataba más de enseñar o instruir, sino de ofrecer condiciones ambientales para que los niños, por medio de la actividad y de su interrelación con el medio, pudieran desarrollarse. María Montessori, al explicar su método, destaca que

La maestra que desea consagrarse a este método educativo, deberá convencerse de esto: no se trata de entregar conocimientos a los niños, ni dimensiones, formas, colores, etc. [...] se trata de reducir nuestro material al nivel de cualquier otro [...] lo que vemos es una radical transferencia de actividad que antes estaba en la maestra y que ahora es confiada, en su mayor parte, al niño. (1965, p. 143).

Esos desplazamientos ocurridos en el campo pedagógico son leídos por muchos autores como procesos más libres y democráticos, que tienen en cuenta las voluntades, los intereses y las necesidades de los niños. Sin embargo, en lugar de eso, podemos argumentar que comprendemos tales prácticas como nuevas estrategias de gobierno mucho más refinadas y sutiles, y, tal vez por eso mismo, mucho más eficaces. Recordemos la fórmula sobre el liberalismo de Foucault (2007a): gobernar menos, para gobernar más.

Esta forma de gobierno que toma el medio ${ }^{4}$ como instancia de operación no se restringe a las técnicas

4 Aunque para el siglo XVIII, periodo de formulación del proyecto liberal, el concepto de medio aún no existía, nos dice Foucault que su "esquema técnico" ya estaba siendo empleado por los urbanistas o los organizadores de los espacios de ciudades como París. Al respecto, el filósofo nos dice que "[...] si bien la noción no existe, yo diría que el esquema técnico de este concepto de medio, la suerte de estructura pragmática que lo perfila de antemano, está presente en el modo como los urbanistas intentan reflejar y modificar el espacio urbano. Los dispositivos de seguridad trabajan, fabrican, organizan, acondicionan un medio aún antes de que la noción se haya constituido y aislado (2006, p. 41). George Canguilhem propone la importancia que ha cobrado el concepto de medio en múltiples campos del saber, al punto de estar "en trance o métodos desarrollados al interior de la escuela. La noción de medio será clave para el desarrollo y la organización de las ciudades a partir de la aparición de lo que Foucault denominó dispositivos de seguridad, los cuales están articulados a la lógica neoliberal, que preferimos llamar, apoyados en Rose (1996), liberalismo avanzado. Esto muestra una serie de desplazamientos señalados por Foucault (2007a, p. 302) cuando dice:

Lo que aparece en el horizonte de un análisis como este no es de ningún modo el ideal o el proyecto de una sociedad exhaustivamente disciplinaria en la que la red legal que aprisiona a los individuos sea relevada y prolongada desde adentro por mecanismos, digamos, normativos. No es tampoco una sociedad en la que se exija el mecanismo de la normalización general y la exclusión de lo no normalizable. En el horizonte de ese análisis tenemos, por el contrario, la imagen, la idea o el tema-programa de una sociedad en la que haya una optimización de los sistemas de diferencia en la que se deje el campo libre a los procesos oscilatorios, en la que se conceda tolerancia a los individuos y las prácticas minoritarias, en la que haya una acción no sobre los participantes del juego, sino sobre las reglas del juego, y, para terminar, en la que haya una intervención que no sea del tipo de la sujeción interna de los individuos, sino de tipo ambiental.

A partir de las ideas planteadas por Foucault, fue posible construir el argumento de que hoy observamos una forma de inclusión de los sujetos que se da por medio de la habitabilidad. Esta establece una relación estrecha con la noción de medio (millie), pues no es más que la cualidad, la particularidad o la condición de aquello que es habitable, del medio. Es decir, se trata de fortalecer, a partir del medio, condiciones para que todos los sujetos puedan circular y habitar el espacio abierto de la ciudad. Sin embargo, no basta con intervenciones en este para garantizar la participación de todos, sino que también son necesarias las inversiones de los sujetos en sus habilidades, competencias, en fin, en la producción de aprendizajes que los tornen capaces de gestionar sus propias vidas en estas ciudades educativas.

La habitabilidad, entonces, surge en el encuentro del sujeto con el ambiente, del habitante con la habitación. Ambos necesitan de intervenciones: el medio, la habitación, y el sujeto, el habitante. A partir de esas intervenciones sobre el sujeto-habitante es que percibimos que, además de la idea de inclusión,

de convertirse en un modo universal y obligatorio de coger la experiencia y existencia de los seres vivientes y casi se podría hablar de su constitución como categoría del pensamiento contemporáneo" (1976, p. 151). 
la noción de aprendizaje también sustenta la condición de habitabilidad. Podemos decir, entonces, que el aprendizaje es hoy la forma de gobierno contemporáneo. Según Noguera (2011, p. 230), "estamos siendo compelidos a comportarnos como aprendices permanentes, que viven en sociedades de aprendizaje o en ciudades educativas".

Sin embargo, ¿quiénes son los habitantes de esas ciudades educativas? 0 mejor, ¿quién debe habitarlas? La respuesta es muy simple: ¡todos! Todos los sujetos deben tener condiciones de habitabilidad en esas ciudades educativas: los pobres, los ricos, los blancos, los negros, los locos, los criminales, los desempleados, los niños de la calle, los niños en vulnerabilidad social, en fin, todos los sujetos, estén clasificados como normales o anormales.

De esta forma, las ciudades educativas o los procesos de aprendizaje permanente se deben desarrollar no solo sobre los sujetos normales, sino también sobre aquellos que Popkewitz (2008) denominó "individuos abyectos". Ellos fueron considerados peligrosos para las sociedades educadas y para los procesos de industrialización y aun de civilización (modernización de la sociedad). Los individuos de las márgenes de la sociedad, aquellos que se encuentran fuera del promedio de normalidad, son los abyectos que necesitaban de la educación para los propósitos de la nacionalización, ciudadanía y otros imperativos de la vida social (actividad, desarrollo, crecimiento). Ese es el proyecto de expansión de la escuela y de los sistemas educativos en función del aprendizaje que debe alcanzar también a los sujetos abyectos, marginales.

La idea de los "abyectos", según Popkewitz, en principio, se refiere a la población de mujeres e inmigrantes de la sociedad norteamericana de las primeras décadas del siglo xx. Según el filósofo, "las reformas progresistas sociales y educativas, en conjunto con las teorías de las ciencias sociales y de la educación, fueron formuladas con el propósito de la inclusión de las poblaciones no civilizadas [...]. Estas reformas incluyeron proyectos de abyección" (2008, p. 35). Para eso, dice Popkewitz, las reformas educativas de mediados del siglo xx incluyeron a los "otros" (mujeres, inmigrantes) como blancos de la educación y, en esa misma racionalidad, fue posible la emergencia del discurso de los derechos de las mujeres, de los niños y, aun, de la democratización de las escuelas, según han dicho Simons, Masschelein y Larrosa (2011) e incluso Ránciere. Lo que nos parece interesante resaltar se refiere al hecho de que la inclusión de esos sujetos abyectos en los procesos de escolarización no se dio por una idea de inclusión benevolente o de acogimiento al otro. Más allá del bien o del mal, la inclusión de ellos funcionó y aún funciona como un proyecto de defensa social, es decir, como una forma de gestionar los riesgos que tales sujetos abyectos pueden causar a la población. Esta gestión se da, prioritariamente, por medio de la educación y de los procesos de aprendizaje a los que los sujetos se vinculan o a los que son sometidos, y a fin de generar mecanismos que faciliten su habitación en la ciudad contemporánea.

En función de tales reformas educativas, el discurso de la igualdad es fundamental como eje de las políticas educativas, motor de la proliferación de pedagogías y horizonte de la formación de profesores. Antes de la Segunda Guerra Mundial (este será un acontecimiento fundamental para el desbloqueo del gobierno liberal y su paso para el neoliberal), los individuos abyectos eran aquellos pertenecientes a grupos minoritarios, como los hemos llamado; después, y con el desarrollo de una racionalidad liberal avanzada, podemos afirmar que los sujetos abyectos comenzaron a ser todos aquellos no escolarizados, pues una de las características más visibles de esta nueva racionalidad consistió, precisamente, en la intensificación y la expansión de la escuela, en nombre del desarrollo y el progreso de "todos", discursos propios de las prácticas liberales avanzadas. Todos aquellos individuos no escolarizados serían abyectos y deberían ser llevados a la escuela. Allí la igualdad se constituyó en regla y no en aspiración.

De esta forma, podemos comprender que el proceso de democratización de la escuela y aun de la educación fueron tácticas, mecanismos y estrategias que funcionaron (y continúan haciéndolo) al servicio de la racionalidad del "liberalismo avanzado" y se constituyeron como puntos de partida para el desarrollo del trabajo pedagógico en las escuelas y también de las ciudades como espacios abiertos para el aprendizaje ${ }^{5}$. De esa forma, podemos decir que la cuestión central no fue solamente la inclusión de las diferencias, sino también la necesidad de inversiones que los sujetos deben realizar en sus vidas. Siendo blancos de la educación, serían blancos de esas estrategias de inversión que les permitirán desarrollar determinadas habilidades para convivir y habitar el espacio abierto de la ciudad.

Contemporáneamente, son muchas las situaciones que nos permiten observar tal movimiento. Todos los sujetos, normales y anormales, requieren estar incluidos en diferentes espacios, teniendo condiciones de participación en la sociedad, el mundo del consumo, el mercado del trabajo y, obviamente, los procesos de

5 Para profundizar esta discusión, véase Fauré (1973) y Delors (1996). 
escolarización. Lo que es posible percibir es que en la lógica neoliberal las inversiones que deben ser hechas en todos los sujetos no son un privilegio de la escuela. Vivimos un momento de pedagogización de lo social en el que la sociedad entera, los diferentes espacios, proyectos y lugares, son espacios educativos que invierten en los sujetos conduciendo sus conductas. Las ciudades, entonces, son espacios privilegiados de los individuos que necesitan de autoinversiones. Así, cada uno necesita para su propia actividad crecimiento y desarrollo de constantes inversiones y autoinversiones, pero de una forma diferente de aquella promovida por la educación de principios del siglo Xx. El crecimiento, la actividad y el desarrollo de finales del siglo xx y hasta hoy tienen que ver con el problema de la sobrevivencia en las grandes ciudades. La idea de la "ciudad educativa" no solo implicó un proceso de "pedagogización general", sino que marcó también su constitución como espacio de aprendizaje, dadas las necesidades de su propia habitabilidad. Esto quiere decir que la ciudad educativa soñada por Fauré et al. (1973) en los años setenta no solo ampliaría las posibilidades de aprendizaje de los sujetos, sino también se constituiría como condición sine que non para ser habitada por ellos.

Entendemos que la ciudad que ofrece condiciones de habitabilidad para todos los individuos (normales o anormales) vive constantemente una paradoja: no necesita más de los individuos que tienen experiencia para enseñar, pues tiene individuos producidos para aprender. Así, en la contemporaneidad observamos un desplazamiento de la "enseñanza hacia el aprendizaje". Las prácticas educativas se han tornado técnicas para el aprendizaje a partir de la igualdad y nuestras luchas se desarrollan en su defensa. Nadie cuestiona el principio de igualdad o se pone en contra de él, pues todos pretendemos practicar la vida bajo el imperativo de la autonomía y la libertad. Ya Noguera (2011), Biesta (2013) y otros han hablado "del nuevo lenguaje del aprendizaje" (p. 34), en el cual han identificado el desplazamiento de la enseñanza hacia el aprendizaje, o la emergencia de la "sociedad del aprendizaje", "ciudad educadora", "aprendizaje a lo largo de la vida" y otros slogans, que tienen como objetivo la habitabilidad de los individuos que son compelidos a buscar bien-estar en la ciudad como espacio insignia del mundo actual.

\section{Referencias}

Bauman, Z. (2005). Vidas desperdiçadas. Rio de Janeiro: Jorge Zahar.

Benvenuto, A. (2006). O surdo e o inaudito. À escuta de Michel Foucault. En J. Gondra y W. Kohan (Eds.), Foucault, 80 anos (pp. 41-58). Belo Horizonte: Autêntica.

Biesta, G. (2013). Para além da aprendizagem. Educação democrática para um futuro humano. São Paulo: Autentica.

Canguilhem, G. (1976). El conocimiento de la vida. Barcelona: Anagrama.

Deleuze, G. (1990). Pos-scriptum sobre as sociedades de controle. En Conversações: 1972-1990 (pp. 219-226). Rio de Janeiro: Ed. 34.

Delors, J. (1996). La educación encierra un tesoro. Santiago: Unesco.

Faurè, E. (1973). Aprender a ser. La educación del futuro. Madrid: Alianza.

Foucault, M. (2001). Defender la sociedad. Buenos Aires: Fondo de Cultura Económica.

Foucault, M. (2006). Seguridad, territorio, población. (Trad. H. Pons). Buenos Aires: Fondo de Cultura Económica.

Foucault, M. (2007a). Nacimiento de la biopolítica. Buenos Aires: Fondo de Cultura Económica.

Foucault, M. (2007b). Historia de la sexualidad 1: la voluntad de saber (31. - ed.) México D.F.: Siglo XXI.

Foucault, M. (2007c). Los anormales. Buenos Aires: Fondo de Cultura Económica.

Foucault, M. (2010). Nietzsche, la genealogía, la historia. Valencia: Pre-textos.

Foucault, M. (2015). Historia de la locura en la época clásica

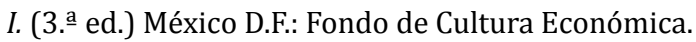

Montessori, M. (1965). Pedagogia científica: a descoberta da criança. Rio de Janeiro: Flamboyant.

Noguera-Ramírez, C. (2011). Pedagogia e governamentalidade ou da modernidade como uma sociedade educativa. São Paulo: Autentica.

Popkewitz, T. (2008). El cosmopolitismo y la era de la reforma escolar. Madrid: Morata. 
Rose, N. (1996). Governing "advanced" liberal democracies. En A. Barry, T. Osborne y N. Rose (Eds.), Foucault and political reason (pp. 37-64). Londres: University of Chicago Press.

Rubio, D. (2017). Historia del aprendizaje como historia de la gubernametalidad neoliberal. una lectura desde el homo œconomicus (tesis inédita de doctorado). Universidad Pedagógica Nacional, Bogotá, Colombia.

Sennett, R. (1994). Carne y piedra: el cuerpo y la ciudad en la civilización occidental. Madrid: Alianza.
Simons, M., Masshelein, J. y Larrosa, J. (Eds.) (2011). Jaques Rancière. La educación pública y la domesticación de la democracia. Buenos Aires: Miño y Dávila.

Veiga-Neto, A. (1996). A ordem das disciplinas (tesis inédita de doctorado). Universidade Federal do Rio Grande do Sul, Porto Alegre, Brasil.

Veyne, P. (1998). Como se escreve a história; Foucault revoluciona a história. Brasília: Editora Universidade de Brasília. 